\title{
Proportion of Low Birth Weight Neonates and Associated Factors Among Mothers Delivered in Wolaita Sodo University Teaching and Referral Hospital, Ethiopia, 2018
}

\author{
Maseresha Assefa Demissie ${ }^{1}$, Lemma Desalegn Hailu ${ }^{1}$, Kelemu Abebe Gelaw ${ }^{2}$ \\ ${ }^{1}$ Department of Nursing, College of Health Science and Medicine, Wolaita Sodo University, Wolaita Sodo, Ethiopia \\ ${ }^{2}$ Department of Midwifery, College of Health Science and Medicine, Wolaita Sodo University, Wolaita Sodo, Ethiopia
}

Email address:

kelemuabebe2014@gmail.com (K. A. Gelaw)

To cite this article:

Maseresha Assefa Demissie, Lemma Desalegn Hailu, Kelemu Abebe Gelaw. Proportion of Low Birth Weight Neonates and Associated Factors Among Mothers Delivered in Wolaita Sodo University Teaching and Referral Hospital, Ethiopia, 2018. American Journal of Pediatrics. Vol. 5, No. 3, 2019, pp. 104-110. doi: 10.11648/j.ajp.20190503.15

Received: May 29, 2019; Accepted: June 29, 2019; Published: July 31, 2019

\begin{abstract}
Low birth weight continues to remain a major public health problem in Ethiopia in contrast to what is observed in many developing countries. The objective of this studt was to asses the proportion of low birth weight and associated factors among mothers who delivered in Wolaita Sodo University Teaching and Referral Hospital,2018. Retrospective cross-sectional study was employed. A total of 295 study participants were participated and selected by using systematic random sampling techniques. The data was entered and cleaned by Epi info version 7.0 and analyzed using SPSS version 20.0. Bivariate and multivariable logistic regression analysis was used.. Significance was declared at $\mathrm{P}<0.05$ in a multivariable logistic interval with $95 \%$ of a confidence interval. The study indicated that $35(12.7 \%)$ of the mothers delivered low birth weight In multivariable analysis; Gestational age $(\mathrm{AOR}=12.203,95 \% \mathrm{CI}=4.695-31.716)$, primi $(\mathrm{AOR}=3.526,95 \% \mathrm{CI}=1.388-8.955)$, pregenancy type $(\mathrm{AOR}=3.491,95 \% \mathrm{CI}=1.063-11.464)$. Antenatal care visit $(\mathrm{AOR}=9.643,95 \% \mathrm{CI}=1.369-67.937)$. The finding of this study was low. The quality of cost-effective focused antenatal care, identifying obstetric complications and addressing them timely is recommended as to the occurrence of low birth weight could be minimized.
\end{abstract}

Keywords: Low Birth Weight, Associated Factors, Southern Ethiopia

\section{Introduction}

World Health Organization (WHO), an infant is considered to be of low birth weight if his/her weight at birth is less than 2500 grams, irrespective of the gestational age of the infant. Classifications of Low Birth Weight (LBW) babies include low birth weight (less than 2500 grams), very low birth weight (less than 1500 grams) and extremely low birth weight (less than 1000 grams) [1].

The average newborn weights about $3500 \mathrm{~g}$. Babies of Low Birth Weight are at increased risk of short and long complicationsof health problems after birth. It is difficult for these babies to breastfeeding, increasing weight and fight infection. Because of their low body fat, it is also difficult for them to stay warm. Most of these low birth weight babies are required intensive neonate care care, usually in a Nursery [2].

Low birth weight is a major determinant of morbidity, mortality, and disability in infancy and childhood and has a long-term impact on health outcomes in adult life [3]. Women with low education, poverty and poor nutritional status who are increased risk of adverse reproductive outcomes including LBW and preterm birth. Therefore important to identify such mothers during pregnancy to determine the level of care and priorities for a referral to centers where reasonable obstetric and neonatal care is available [4]. The neonates of adult mothers are also more likely to have LBW with the risk of long-term effects [5]. Incidence of LBW ranged from $6 \%$ to $18 \%$ across the globe with Sub-Saharan Africa accounting $13 \%$ to $15 \%$ [6]. In Kenya, studies show that the proportion of low birth weight was $11.2 \%$ [7]. The prevalence of low birth weight vary from region to region in Ethiopia. For example, study conducted in Jimma reported that proportion of low birth weight was $22.5 \%$ [8] and a recent study in Gondar showed $17.1 \%$. In 
Ethiopia, there is limited information on birth weight distribution. Especially, there is no adequate information on the proportion and associated factors of low birth weight in the study setting. Therefore, this study aimed to assess the proportion and associated factors of low birth weight in Wolaita Sodo University Teaching and Referral Hospital.

The main purpose of this study was to provide baseline data on the proportion of low birth weight in the study setting and to identify possible factors for low birth weight were be help to inform the health authorities about the local associated factors for LBW.

\section{Methods and Materials}

\subsection{Study Area}

This study was done at Wolaita Sodo University Teaching Referral Hospital which is found $396 \mathrm{~km}$ south from Addis Ababa and $165 \mathrm{~km}$ far from Hawassa, South Ethiopia.

The hospital is serving about three million people in the catchment area and people including in the neighboring zones. About 80,000 people visit Output patient department annually. The Hospital has 250 beds and 423 staff including specialists, general practitioners, X-ray technician, health officers, nurses, Midwives, laboratory professionals, pharmacists, druggists, and Administrative staffs. Out of these, 60beds inward, of Obstetrics / Gynaecology which 10 beds are in labor ward and 50 beds, are in Gynecology ward.

Obstetrics\& Gynaecology department has its building with set up of Neonatal Intensive Care Unit, output patient department, Kangaroo mother care unit, Emergency output patient department, Antenatal care, Family planning, Laboratory, Delivery service unit, postnatal care unit, Obstetrics ward, Gynaecology ward, Operation room, Post anaesthetic care unit, and recovery unit. Regarding staff, the department has three Gynaecologists, 32 Midwives (BSC \& Diploma) and there are also residents, IESO students and medical Interns currently practicing.

\subsection{Study Design and Period}

Institution based Retrospective cross-sectional study was conducted at Wolaita Sodo University Teaching \& Referral Hospital, SNNPR, and Ethiopia among women delivering neonates from July 1, 2017, to 30 July 2018 G.C.

\subsection{Study Population}

All selected mothers who delivered at WSUTRH from July 1, 2017, to July 30, 2018, G.C.

\subsection{Sample Size Determination and Sampling Procedure}

The sample size for this particular study was calculated using a formula for a single population proportion considering the following assumptions.

Prevalence of low birth weight and its Associated Factors among Mothers Delivered in Jimma University Specialized Teaching and Referral Hospital, Jimma Zone,
Oromia Regional State, South West Ethiopia is found to be $22.5 \%$ [13], 95\% CI, 5\% marginal error, and 10\% nonresponse rate.

$(\mathrm{p}=22.5)$ was substituted in the following single population proportion formula.

$$
\begin{gathered}
\mathrm{n}=\frac{(\mathrm{Z} \alpha / 2)^{2} \mathrm{p}(1-\mathrm{p})}{\mathrm{d} 2} \\
\mathrm{n}=\frac{(1.96)^{2}(0.225)(1-0.225)}{0.0025}
\end{gathered}
$$

$\mathrm{Z}$ o $/ 2=$ the value of standard normal distribution corresponding to a significant level of alpha.

$\mathrm{p}=$ proportion of the problem $(22.5 \%)$

$\mathrm{d}=$ an absolute precision (margin of error 5\%). By adding $10 \%$ non-respondent rate for incomplete data, the required total sample sizes for the study was $27+268=295$.

The Delivery register was used to obtain the sampling frame. Systematic sampling was used to enroll women in the study. Approximately 4536 women deliver at the hospital monthly, and since the study was conducted over four months, the total number of women who delivered over the months was expected to be 378 . With a calculated sample size of 295 , therefore the sampling interval was, $k=N / n$; were $\mathrm{k}=$ constant value,

$\mathrm{N}=$ Source population $=4536, \mathrm{n}=$ sample sizes $=295$ $4536 / 295=15$.

The interval was 15 . The first sampling was chosen by lottery sampling method which start point was 10 using a random number delivery book, every $15^{\text {th }}$ delivery was selected until the desired sample size was achieved.

\subsection{Data Collection Techniques}

Information obtained from previous studies on low birth weight and other obstetrics and gynecology sites was used in addition to other variables, to design a structured questionnaire for data collection. Data was collect by using structured questionnaire from medical records of mothers, admission history, labour follow up sheet, delivery summary, antenatal care follows up sheet, Neonatal register book and Postnatal registration book used to get information for the study variables. This includes socio-demographic, Obstetrics factors, medical and health promotion factors and newborn factors. Beside principal investigator, there was one supervisor. The supervisor and data collectors were trained for a day on basic principles of data collection, on the questionnaire and how to do other related procedures during data collection by the principal investigator. Additional training on data completeness, cross-checking and correction actions was given to the supervisor. Accordingly, the supervisor continuously followed and supervised data collectors by collecting and cross-checking the completeness of questionnaires were received from data collectors and took corrective measures accordingly. And also reported and communicated with the principal investigator daily throughout the data collection period. 


\subsection{Exclusions}

Medical records that have no explanation for gestational age were excluded.

Charts that did not have complete information relevant for the study were excluded.

\subsection{Variables}

\subsubsection{Dependent Variables}

Low Birthweight.

\subsubsection{Independent Variables}

\section{Socio-demographic factors}

Age, residence, Mother's education level, Partner's education level, employment status of mother occupation, Religious background, Marital status, Smoking.

\section{Obstetrics Factors}

Age at first birth, number of previous births, Gestational age, number of pregnancies, preceding birth interval, last baby weight, bad obstetric history, previous history of low birth weight or prematurity, previous surgery on uterus.

\section{Maternal health care}

Antenatal care (number of ANC visits).

Mother's health status

Sexually transmitted infection, HIV/AIDS, Syphilis and chronic conditions like, hypertension, malaria attacks, maternal disability, maternal stress....

\section{Maternal nutritional status}

Anemia, underweight (maternal weight, nutrient deficiency e.g. Iron and Foliated supplementation.

Operational definitions

Parity: Number of pregnancies carried to viability and delivered.

Multiparous: Has had two or more deliveries that were carried to be viability.

Term: gestational age between 37-41weeks and 6 days.

Pre term: gestational age of $<37$ weeks.

Low birth weight: birth weight $<2500 \mathrm{gm}$.

Normal birth weight: birth weight 2500gm-3999gm.

\subsection{Data Quality and Control and Data Analysis Procedures}

The questionnaire was pre-test in around five percent of study subjects in WSTRH by taking Medical records of mothers who give birth from out of July 2017 to July, 2018G.C and was reviewed. To maintain data quality, giving trained for data collectors by the investigator's supervisor. The questionnaire was pre-tested to check for the accuracy of responses and appropriateness of the data collection tool. Completeness and consistency by the principal investigator and supervisors on daily bases during data collection time.

The data was checked and entered using Epic-Info version 7.0. It was cleaned and edited accordingly and exported to SPSS Version 20.0 and checked for missing values before analysis. Descriptive statistics using the measure of central tendency and dispersion, frequencies, proportions, and diagrams were used to check its distribution and describe the study population with relevant variables.

\subsection{Ethical Considerations}

An official letter was written from Wolaita Sodo University College of Health Science and Medicine, Department of Neonatal and Paediatrics Nursing to Wolaita Sodo UniversityTeaching and Referral hospital chief clinical director office and to get permission from WSUTRH before collecting data from clients' charts.

\section{Results}

\subsection{Socio-demographic Characteristics}

The purpose of the study is to examine the associated factors that contribute to low birth weight. Data were collected from hospital records of mothers who gave birth to babies in one year period of time. Therefore, availability sampling was used for the study. The data collected from the chart reviews were analyzed using descriptive statistics (frequencies and percentages).

A total of 295 records of women gave birth in the study setting were incorporated in the study. Of these, $19(6.4 \%)$ in respondents were excluded which were invalid due to different reasons or errors in the review were intolerable. Therefore, from a total of 276 (93.5\%) charts reviewed and included in the current study.

Regarding socio-demographic characteristics of mothers who given birth at the study setting about 60(21.7\%) were aged less than 20 years, $173(62.7 \%)$ were aged between 20 35 years and $43(15.6 \%)$ was aged above 35 years old. The majority $208(75.4 \%)$ of reviewed charts identified as Wolaita in their ethnicity whereas the list $68(24.6 \%)$ of them were others. More than half of the presence of women who give birth at the Hospital were from out of Sodo town whereas the rest resided of Sodo town. (See table 1)

Table 1. Distribution of mothers by Socio-demographic characteristics who delivered at WSUTRH, from July 1, 2017, to July 30, 2018 G.C, Sothern, Ethiopia, $2018(N=276)$.

\begin{tabular}{llll}
\hline Variables & & Frequency $(\mathbf{N})$ & Percent (\%) \\
\hline \multirow{2}{*}{ Maternal Age } & $<$ 20 Years & 60 & 21.7 \\
& 20-35 Years & 173 & 62.7 \\
& $>$ 35 Years & 43 & 15.6 \\
& Wolaita & 208 & 75.4 \\
Ethnicity & Gamo & 30 & 10.9 \\
& Kambata & 25 & 9.1 \\
Residence & Others & 13 & 4.7 \\
$\mathrm{n}=208$ & Sodo Town & 129 & 46.7 \\
\hline
\end{tabular}

\subsection{Obstetrics Factors}

Regarding Obstetric characteristics, 268(97.1 \% for women who have a history of Antennal care follow-up. From these, more than half mothers ANC follow up at WSUTRH, $86(31.2 \%)$ were followed up at other health facilities and the rest of mothers were no evidenced for ANC follow up. Among ANC followers, more than half of the mothers were 
multiparity and 114(41.3) were the first time delivery. Ninety-seven percent of mothers had received nutritional counseling. Even though, only 104 (37.7\%) were exposure of Iron and folic acids during pregnancy.

From 276 mothers, more than eighty-five percent of the mothers were $37-42$ weeks gestation at the time of delivery and the rest of $35(12.7 \%)$ were preterm. Deliveries that completed by spontaneous vaginal deliveries were $221(80.1 \%)$ and cesarean section 55(19.9\%).

The majority of the deliveries $250(90.6 \%)$ were single neonatal deliveries, 26 (9.4\%) were Multiple deliveries. Obstetric complications were present in about $7.2 \%$ of women having a history of abortion was recorded in $4.7 \%$ of participant's pregnancies. From the total, current pregnancy $263(95.3 \%)$ were planned and $13(4.7 \%)$ were unplanned.

From total newborn, $131(47.5 \%)$ male sex, 145(52.5\%) females. From 276 mothers, the majority of them did not experience bleeding during pregnancy 269(97.5\%) and only $7(2.5 \%)$ experienced bleeding during pregnancy. Nearly ninety-seven percents of mothers were not reporting having had a low birth weight baby in their previous pregnancy. Only 5(3.09\%) mothers were reported having had reported a history of low birth weight.

From the total, most of the mothers' weighted $50 \mathrm{~kg}$ and above $249(90.2 \%)$ and $5(1.8 \%)$ of those mothers' weights were less than $50 \mathrm{~kg}$ whereas the rest of mothers were not recorded.

From total delivered, two hundred forty-one $(87.3 \%)$ were weighed above 2500grams and 35 (12.7\%) were weighed less than 2500 grams. The prevalence of low birth was $35(12.7 \%)$. (See from table 2 below.)

Table 2. Obstetric factors of Mothers who given birth at WSUTRH from July 2017 to July 2018 G.C. Southern, Ethiopia, 2018 (N=276).

\begin{tabular}{|c|c|c|c|}
\hline Variables & Categories & Frequency (N) & Percent $(\%)$ \\
\hline \multirow{2}{*}{ ANC follow-up } & Yes & 270 & 97.8 \\
\hline & No & 6 & 2.2 \\
\hline \multirow{4}{*}{ Place ANC follow up } & WSUTRH the hospital & 184 & 66.7 \\
\hline & other health facilities & 86 & 31.2 \\
\hline & no recorded evidence & 6 & 2.2 \\
\hline & Total & 276 & 100.0 \\
\hline Parity & Primiparous & 114 & 41.3 \\
\hline \multirow{2}{*}{ Nutritional counseling } & Yes & 268 & 97.1 \\
\hline & No & 8 & 2.9 \\
\hline \multirow{2}{*}{ Iron and foliate acid intake } & Yes & 104 & 37.7 \\
\hline & No & 172 & 62.3 \\
\hline \multirow{3}{*}{ Gestational age } & Preterm (<37 Weeks) & 35 & 12.7 \\
\hline & Term (37 to 42 weeks) & 241 & 87.3 \\
\hline & Total & 276 & 100.0 \\
\hline \multirow[t]{2}{*}{ Mode of delivery } & Cesarean section & 55 & 19.9 \\
\hline & Total & 276 & 100.0 \\
\hline \multirow{3}{*}{ The pregnancy type } & Single & 250 & 90.6 \\
\hline & Multiple & 26 & 9.4 \\
\hline & Total & 276 & 100.0 \\
\hline \multirow{2}{*}{ Medical complications } & Yes & 39 & 14.1 \\
\hline & No & 237 & 85.9 \\
\hline \multirow{3}{*}{ History of abortions } & Yes & 13 & 4.7 \\
\hline & No & 263 & 95.3 \\
\hline & Total & 276 & 100.0 \\
\hline \multirow{2}{*}{ Current pregnancy } & Planned & 263 & 95.3 \\
\hline & Unplanned & 13 & 4.7 \\
\hline \multirow{2}{*}{ Sex of baby } & Male & 131 & 47.5 \\
\hline & Female & 145 & 52.5 \\
\hline \multirow{2}{*}{ Vaginal bleeding during pregnancy } & Yes & 7 & 2.5 \\
\hline & No & 269 & 97.5 \\
\hline \multirow{3}{*}{ Maternal weight } & Less $50 \mathrm{~kg}$ & 5 & 1.8 \\
\hline & $\geq 50 \mathrm{~kg}$ & 249 & 90.2 \\
\hline & Not recorded/unknown & 22 & 8.0 \\
\hline \multirow{2}{*}{ Weight of newborn } & Normal birth weight & 241 & 87.3 \\
\hline & Low birth weight & 35 & 12.7 \\
\hline
\end{tabular}

\subsection{Antenatal Care Visits}

From 276 mothers, more than half mothers who given birth at in Wolaita Sodo university teaching and referral hospital. Mother has Antennal care follow up at the hospital were $66.7 \%$ of the reviewed charts. $97.8 \%$ mothers had a history of antenatal care follow up, of whom (50.7\%) having first Antennal care visits, 84 (30.4\%) had 2-3 follow up and $46(16.7 \%)$ had fourth and greater than four visits during the 
last pregnancy (figure 1).

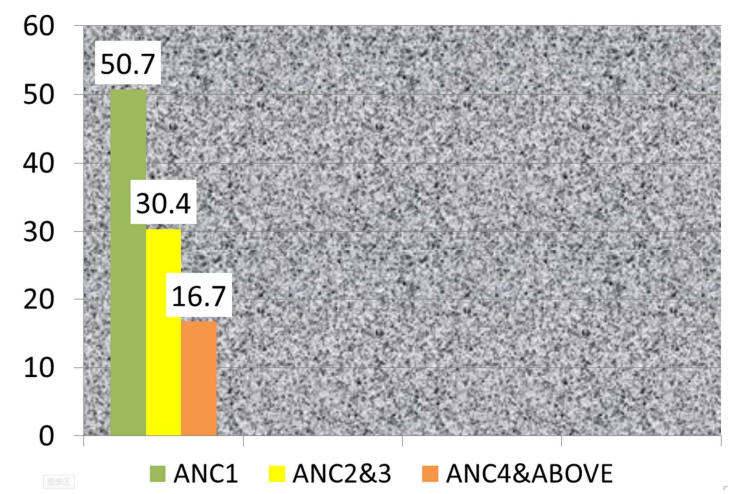

Figure 1. Mothers who visit ANC follow up at WSUTRH from July 1, 2017, to July 30, 2018, G.C. Southern, Ethiopia, 2018.

\subsection{Maternal Obstetric and Medical Disorders}

Two hundred seventy-six mothers were assessed obstetric and medical illnesses. Among women with obstetric problems and medical illness, 10(3.6\%) had preeclampsia/eclampsia was the first medical disorders in these observations. Others, $8(2.9 \%)$ sexually transmitted infection/UTI, 2(0.7\%) were HIV/AIDS positive, $2(0.7 \%)$ had been tested hepatics $\mathrm{C}$ positive, $3(1.1 \%)$ had Hepatitis $\mathrm{B}+\mathrm{Ve}, \quad 4(1.4 \%)$ had anaemia, $4(1.4 \%)$ had chronic hypertension and 6(2.2) were other disorders.

\subsection{Associated Factors of Low Birth Weight}

To see independent predictors of hospital delivery service, those factors found associated on a multivariable analysis involving all associated variables was performed to identify independent predictors of low birth weight. Consequently, gestational age, parity, pregnancy type and a woman who had ANC visit were independently showed significant association. The details are summarized on the table below.

A woman whose Gestational age was preterm had more likely to deliver low birth weight than those whose GA was a term. Women whose GA preterm were twelve times more likely to deliver low birth weight than women whos GA were a term $(\mathrm{AOR}=12.203,95 \% \mathrm{CI}=4.695-31.716)$.

Moreover, mothers who were primipara become three and half times more likely to deliver low birth weight than those who were multipara $(\mathrm{AOR}=3.526,95 \% \mathrm{CI}=1.388-8.955)$.

From the total of respondents, mothers who had multiple pregnancies were three and half times more likely to deliver low birth weight than those who had single pregnancy (AOR=3.491, 95\% CI =1.063-11.464).

Women who had no ANC visit were more likely to deliver low birth weight than that of who had ANC visit. Mothers who had no ANC visit were nine and half times more likely to deliver low birth weight than women who had ANC visit $(\mathrm{AOR}=9.643,95 \% \mathrm{CI}=1.369-67.937)$.

Table 3. Bivariate and multivariable analysis results of mothers who gave birth in WSTRH from July 1, 2017, to July 30, 2018, G.C, SNNPR, Ethiopia, 2018.

\begin{tabular}{|c|c|c|c|c|c|c|c|c|c|c|}
\hline \multirow{2}{*}{ Variables } & \multirow{2}{*}{ Category } & \multirow{2}{*}{\multicolumn{2}{|c|}{$\begin{array}{l}\text { Birth weight } \\
\text { Normal (\%) LBW (\%) }\end{array}$}} & \multirow{2}{*}{ COR } & \multicolumn{2}{|c|}{$95 \%$ CI } & \multirow{2}{*}{ AOR } & \multicolumn{2}{|c|}{$95 \%$ CI } & \multirow{2}{*}{ P value } \\
\hline & & & & & Upper & Lower & & Upper & Lower & \\
\hline \multirow{2}{*}{ Gestational age } & Pre term & $20(8.3)$ & $15(42.9)$ & 8.3 & 3.68 & 18.64 & 12.203 & 4.695 & 31.716 & 0.000 \\
\hline & Term & $221(91.7)$ & $20(57.1)$ & 1 & & & 1.00 & & & \\
\hline \multirow{2}{*}{ Parity } & Primi para & $97(40.2)$ & $17(48.6)$ & 0.004 & 3.4 & 10.4 & 3.526 & 1.388 & 8.955 & 0.008 \\
\hline & Multipara & $144(59.8)$ & $18(51.4)$ & & & & 1.00 & & & \\
\hline \multirow{2}{*}{ Pregnancy type } & Single & $223(92.5)$ & $27(77.1)$ & 1 & & & 1.00 & & & \\
\hline & Multiple & $18(7.5)$ & $8(22.9)$ & 3.6 & 1.45 & 9.24 & 3.491 & 1.063 & 11.464 & 0.039 \\
\hline
\end{tabular}

\section{Discussion}

The proportion of low birth weight in this study was $12.7 \%$. This Proportion was higher 7\% documented in the developed countries [9]. Similarly, it was higher (9.1\%) which was retrospective study conducted in Mother and Child Health clinics in three facilities in Korowai district, Tanzania [10]. Based on Ethiopian Demographic and Health Survey reports the proportion of LBW in Ethiopia estimated to be $11 \%$ [11]. The proportion was lower than $23 \%$ documented in Central Africa [12]. Also, it was lower 22.5\% Jimma zone, Southwest Ethiopia [13]. But this result was similar to studies documented in Sudan [14]. Also, the study was nearly similar research conducted in all hospitals Addis Ababa indicated an LBW rate of [15]. Also, this difference may be the studies setting and sample sizes.

This study reveals that majority of the women delivering in Wolaita Sodo Teaching and Referral Hospital during the study period were aged 20-35 years. This finding was similar with findings documented elsewhere [16].
According to study coducted Kenya study, more than eight five percent of the mothers were from Rural residence [10]. A similar study conducted in Jimma zone, Southwest Ethiopia found 65 percent of the mothers attended the maternity facilities to be of rural residence [13]. In our study, More than half of the percent of mothers who gave birth at the Hospital were from rural residents than urban to similar to our findings.

In this study, some factors were found to be significantly associated with low birth weight on bivariate and multivariate analysis. They included having, gestational age (Preterm), parity, pregnancy type and a woman who had ANC visits.

In our study showed that preterm was 12 times more likely to be low birth weight compared to normal ones. A similar, study conducted in Tigray and Gondar University referral hospital the preterm baby was factors associated with LBW [17]. Study conducted in Pakistan showed that Preterm birth increased the risk of low birth weight six times compared to term babies [18]. The differences of this study from study conducted in Pakistan may be Sociodemographic status.

In our study, a lack of focused antennal care follows was 
nine times increasing low birth weight compared to focused antennal care followers. Similarly, Crossectional study conducted Jimma Zone, Southwest Ethiopia showed that Lack/infrequent of antenatal care follow-up was associated with LBW [13].

\section{Conclusion and Recommendation}

The proportion of low birth weight is low in this study. The associated factors of low birth weight were gestational age (preterm), parity, pregnancy type and a woman who had ANC visits may contribute to the occurrence of low birth weight. Therefore, the quality of cost-effective focused antenatal care identifying the medical illnesses as well as obstetric complications and addressing them timely is recommended as to the occurrence of low birth weight could be minimized.

\section{Abbreviations}

ANC; Antenatal care, EDHS; Ethiopian Demographic and Health Survey, HIV Human Immune Virus, HTN; Hypertension, LB; Low Birth Weight, OBS \& GYN Obstetrics \& Gynaecology, SNNPR; Southern Nations, Nationalities, and Peoples Region, SPSS; Statistical Package for Social Sciences, UNICEF; United Nations Children's Fund WHO; World Health Organizations, WSUTRH; Wolaita Sodo University Teaching and Referral Hospital.

\section{Declaration}

Ethics approval and consent to participant

Permission was obtained from Wolaita Sodo University, College of Health Sciences, and Department of Nursing. Also, the letter of permission was secured fro Wolaita Sodo University referral hospital management. Confidentiality of information was maintained.

\section{Consent for Publication}

Not applicable.

\section{Availability of Data and Materials}

The data that support the findings of this study are available but some restrictions may apply to the availability of these data as there are some sensitive issues. However, data are available from the corresponding authors upon reasonable request.

\section{Competing Interests}

The authors declare that they have no competing interests.

\section{Funding}

Wolaita Sodo University.

\section{Authors' Contributions}

MA was involved in the conception, design, analysis, interpretation, report, manuscript writing, design, analysis, interpretation and report writing. LD and KA were involved in design, analysis, and interpretation of the data. Both authors read and approved the final manuscript.

\section{Acknowledgements}

We would like to express our deepest heartfelt thanks to Wolaita Sodo University for allowing the conduct of this study. Our special thanks go to Wolaita Sodo University referral hospital staffs for their support during the data collection process.

\section{Authors' Information}

Masresha Assefa and Lemma Desalegn Hailu is a lecturer in the Department of Nursing, College of Health Science and Medicine, Wolaita Sodo University, Wolaita Sodo, Ethiopia.

Kelemu Abebe Gelaw is a lecturer in the Department of Midwifery, College of Health Science and Medicine, Wolaita Sodo University, Wolaita Sodo, Ethiopia.

\section{References}

[1] World Health Organization International statistical classification of diseases and related health problems, tenth revision (1992). Geneva: World Health Organization.

[2] Back, W. (2010). Low Birth Weight. March of Dimes, Professionals and Researchers Available at; HTTP Accessed on February 2010.

[3] WHO (2011). Preventing early pregnancy and poor reproductive outcomes among adolescents in developing countries: what the evidence says. Geneva, Switzerland: WHO, Maternal N, Child and Adolescent Health.

[4] Negara, D. K. 1998. NC. "Predictors of Low Birth at the Community Level." East Africa Medical J. 75 (5): 296-9.

[5] Megabiaw BM, Zelalem M, Mohammed N. Incidence and correlates of low birth weight at a referral hospital in North West Ethiopia. Pan Africa Med J. 2012; 4: 112.

[6] Siza J. E. (2008). Risk factors associated with a low birth weight of neonates among pregnant women attending a referral hospital in northern Tanzania. Tanzania Journal of Health Research, 10 (1), 18.

[7] Mmbando, B. P., ColeLewis, H., Sembuche, S., Kamugisha, M. L., Theander, T., Lusingu, J. P. Lemnge, M. M. (2008). Risk factors for low birth-weight in areas with varying malaria transmission in Korogwe, Tanzania: Implications for malaria control. Tanzania Journal of Health Research. 10 (3), 137-43.

[8] Berihun, M. Z., Meseret, Z. \& Nuru, M. (2012). Incidence and correlates of low birth weight at a referral hospital in North West Ethiopia. Pan African Medical Journal, 12 (4). 
[9] United Nations Children's Fund and World Health Organization, (2004). Low Birthweight: Country, regional and global estimates. UNICEF, New York.).

[10] Bodeau-Livinec, F., Briand V., Berger J., Xiong Xu., Massoud bodji A., Day K. P... Cot M. (2011). Maternal Anaemia in Benin: Prevalence, Risk Factors, and Association with Low Birth Weight. American Journal of Tropical Medicine and Hygiene. 85 (3), 414420. doi: 10.4269/ajtmh.2011.10-59.

[11] Central Statistical Agency of Ethiopia and ICF International. Ethiopian Demographic and Health Survey 2011. Addis Ababa, Ethiopia, and Calverton, Maryland, USA: Central Statistical Agency and ICF International 2012.

[12] Grace Kwamboka Mogire (2015). Factors Associated with Low Birth Weight deliveries in Pumwani Maternity Hospital, Nairobi- Kenya. JORNAL HEALTH RESEARCH; 79 (4).

[13] Tema T (2006). Prevalence And Determinants of Low Birth Weight in Jimma Zone, Southwest Ethiopia. East African Medical Journal 83.

[14] Elshibly, E. M., and Schmalisch, G. 2008). The effect of maternal anthropometric characteristics and social factors on gestational age and birth weight in Sudanese newborn infants. BMC Public Health, doi: 10.1186/1471-2458-8-244.

[15] Gebremedhin M, Ambaw F, and Admassu E, Berhane H (2015) maternal associated factors of low birth weight: a hospital-based cross-sectional mixed study in Tigray Northern Ethiopia. BMC Pregnancy Childbirth 15.

[16] Demelash H, Motbainor A, Nigatu D, Gashaw K, and Melese A (2015). Risk factors for low birth weight in Bale zone hospitals, South-East Ethiopia: a case-control study. BMC Pregnancy Childbirth 15.

[17] Adane AA, Ayele TA, Ararsa LG, Bitew BD Zeleke BM
(2014). Adverse birth outcomes among deliveries at Gondar University Hospital, Northwest Ethiopia. Childbirth 14.

[18] Badshah S, Mason L, McKelvie K, Payne R, Lisboa PJ. Risk factors for low birthweight in the public hospitals at Peshawar, NWFP-Pakistan. BMC Public Health 2008; 8: 197-198.

[19] Fikre E, Aklilu M. Change in birth-weight of HospitalDelivered neonate in Addis Ababa, Ethiopia. J Health Dev. 2000; 14: 169-72.

[20] Kenya National Bureau of Statistics (Nairobi). (2010). Kenya Demographic and Health Survey 2008-09. Kenya National Bureau of Statistics.

[21] Luke B, Keith LG. The contribution of singletons, twins, and triplets to low birth weight, infant mortality, and handicap in the United States. J Reprod Med 1992; 37: 661-6.

[22] Magadi, M., Diamond, I. Madise, N \& Smith, P. (2004). Pathways of the determinants of Alemu T, Umeta M (2015). Prevalence and Determinants of Small Size Babies in Ethiopia: Results from in-depth Analyses of the Ethiopian demographic health survey-2011. family medicine \& Medical Science Research 4.

[23] Oni, G. A. Twins and their birth sizes in a Nigerian community. East Afr. Med. J. 1983; 60: 492-497.

[24] Perera, F. P., Rauh V., Tsai W. Y., Kinney P., Camann D., Barr D.,...\& Wyatt R. M. (2003). Effects of Trans-placental Exposure to Environmental Pollutants on Birth Outcomes in a Multiethnic Population. Environmental Health Perspectives, 111 (2), 2015

[25] Ricketts, S. A., Murray, E. K. and Schwal Berg, R. (2005). Reducing Low Birthweight by Resolving Risks: Results from Colorado's Prenatal Plus Program. American Journal of Public Health, 95 (11): 1952-1957. 